\title{
Visual Activation Positron Emission Tomography for Presurgical Evaluation of Occipital Lobe Epilepsy
}

\author{
-Case Report-
}

\author{
Hideyuki NAKAMA, Satoru OHTOMO, Taisuke OTSUKI, Yuu KaneKO, \\ Takashi OHNISHI*, and Hiroshi MATSUDA*
}

\begin{abstract}
Departments of Neurosurgery and *Radiology, National Center Hospital for Mental, Nervous and Muscular Disorders, National Center of Neurology and Psychiatry, Kodaira, Tokyo
\end{abstract}

\begin{abstract}
A 14-year-old boy suffered from daily epileptic seizures originating from the left polymicrogyric occipital cortex. Visual activation positron emission tomography (PET) was used to map the function of the occipital cortex presurgically. Loss of visual function in the left occipital cortex was suggested by both visual activation PET and electrical cortical stimulation. Left occipital lobectomy resulted in a completely seizure-free status without deterioration in the visual function. Preoperative evaluation of the visual function in the epileptogenic occipital cortex by activation studies using PET or functional magnetic resonance imaging is the key to the successful surgical treatment of occipital lobe epilepsy.
\end{abstract}

Key words: occipital lobe epilepsy, functional mapping, positron emission tomography, electrical stimulation, visual cortex

\section{Introduction}

Occipital lobe epilepsy is not common and the exact prevalence is unknown. Only $1 \%$ of all patients in one series had undergone occipital lobe surgery for epilepsy. ${ }^{11,12)}$ Several surgical approaches including lesionectomy and occipital lobectomy are used since a uniform surgical approach is difficult to establish for occipital lobe epilepsy because of the variable seizure manifestations, seizure spread patterns, and difficulty in electroencephalography (EEG) localization of the epileptic focus. ${ }^{1,2,12,15)}$ Careful preoperative examination of the visual function of the occipital lobes is essential. Patient selection for surgical treatment is easier if there is a preoperative visual field defect. However, in patients with no preoperative visual field defect or with a smaller defect, the operative indication is difficult because of the high incidence of postoperative complete visual field defect.

Functional mapping of the occipital cortex in patients with occipital lobe epilepsy provides important diagnostic and prognostic information that enhances the chances for successful treatment. Conventional methods such as electrical stimulation and

Received August 15, 2001; Accepted May 31, 2002 evoked potentials have been applied to obtain functional maps of the primary visual cortex and associated areas. In recent years, there have been significant developments in noninvasive techniques for brain mapping, including positron emission tomography (PET) and functional magnetic resonance (MR) imaging. These new techniques have enabled accurate noninvasive mapping of the human visual cortex.

We report a case of occipital lobe epilepsy and illustrate the applicability of visual activation PET for preoperative assessment and surgical planning.

\section{Methods}

\section{Visual activation PET}

PET was performed using a scanner (CTI/Siemens HR + PET scanner; CTI, Knoxville, Tenn., U.S.A.) that produces 47 slices at an intrinsic resolution of $3.8 \times 3.8 \times 4.7 \mathrm{~mm}$. A soft head pad was fitted on the patient to minimize head movement. Intravenous bolus injections of $7 \mathrm{mCi}(259 \mathrm{MBq})$ of $\mathrm{H}_{2}{ }^{15} \mathrm{O}$ were given, and a total of seven scans were performed alternating the resting and visual activation states at intervals of 10 minutes. Visual activation was achieved by binocular photic stimulation of 1.0-Hz flashing red lights administered through spe- 
cial goggles (Nihon Kohden Corporation, Tokyo). Stimulus presentation and task performance began 20 seconds prior to the intravenous bolus injection of the tracer and image acquisition began at the time of injection and continued for 90 seconds. Data were analyzed on a Sun Sparc 20 workstation (Sun Computers Japan Inc., Tokyo) using statistical parametric mapping (SPM) software (SPM 96; Welcome Department of Cognitive Neurology, London, U.K.).6) Prior to image analysis, all scans were realigned to the first scan on a voxel-by-voxel basis using the SPM software. The scans were transformed into stereotactic space using both linear and nonlinear three-dimensional transformation methods to allow intrasubject averaging. The stereotactically normalized scans contained 68 planes (voxel size $2 \times 2 \times$ $2 \mathrm{~mm}$ ) corresponding to the atlas of Talairach and Tournoux. ${ }^{13)}$ Smoothing with a 12 -mm gaussian kernel produced a resolution of $17 \times 17 \times 20 \mathrm{~mm}$ for the final image.

The adjusted regional cerebral blood flow (CBF) images (normalization of global CBF for each scan to $50 \mathrm{ml} / 100 \mathrm{~g} / \mathrm{min}$ with analysis of covariance) during the visual activation and resting states were analyzed and compared. After the appropriate design matrix was specified, the conditions were estimated according to the general linear model at each voxel. These analyses generated (SPM $\{t\})$ maps that were subsequently transformed to the unit normal distribution $\operatorname{SPM}\{Z\}$. The exact level of significance of volumes of difference between conditions was characterized based on peak amplitude. Clusters of voxels with a peak Z-score of $>1.96$ (threshold $p<$ $0.05)$ were considered to show a significant difference. A corrected $p$ value of $<0.05$ was used as the statistical cluster threshold.

\section{Electrical stimulation}

Electrical stimulation mapping was performed via implanted subdural grid electrodes. Stimulation was delivered in a bipolar mode through electrodes separated by $10 \mathrm{~mm}$, using 3 -second trains of $100-\mathrm{Hz}, 0.3-$ msec, biphasic square-wave pulses from a constant current stimulator. The amplitude was increased in increments of $1 \mathrm{~V}$ and the maximal amplitude used was $6 \mathrm{~V}$. The amplitude was never increased above the threshold for afterdischarges.

\section{Case Report}

A 14-year-old right-handed boy had a complicated birth, with probable anoxia. He had suffered medically intractable daily complex partial seizures since 7 years of age. Typically, his seizure began with motionless staring, followed by eye and head devia-
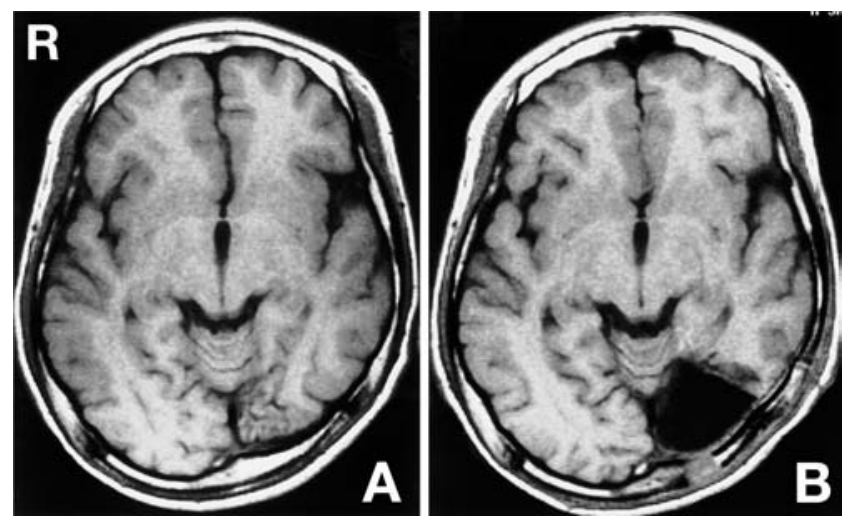

Fig. 1 A: Preoperative magnetic resonance (MR) image showing left occipital ulegyria. B: MR image after the left occipital lobectomy.

tion to the left with or without secondary generalized tonic/clonic convulsion. Interictal scalp EEG showed continuous epileptiform discharges consisting of spikes, sharp waves, and sharp slow wave complexes from the bilateral occipitoparietal and posterior temporal regions, and ictal scalp EEG with video monitoring showed fast waves from the bilateral occipital lobes. MR imaging revealed left occipital polymicrogyria (Fig. 1A). Interictal single photon emission computed tomography (SPECT) showed hypoperfusion in the left occipital and posterior temporal regions, and ictal SPECT showed hyperperfusion in the left medial occipital cortex (Fig. 2A, B). Neurological examination found no deficits, but right homonymous hemianopsia was suspected based on results of the confrontation test. Ophthalmological assessment showed he could not focus his vision because of nystagmus-like involuntary ocular movements and could not perform conventional perimetry. Moreover, visual evoked potentials could not be obtained because of noncooperation of the patient.

The clinical problem was to identify any functional visual cortex in the left occipital lobe, since this was likely to be resected during surgery. Therefore, functional mapping of the left occipital cortex was planned using visual activation PET and cortical stimulation. Informed consent was received for the PET study in accordance with the guidelines of the hospital. Visual activation PET coregistered to the normalized brain MR imaging showed neither activation nor deactivation in the left occipital cortex (Fig. 2C). Subdural grid electrodes were placed over the left occipital cortex including the mesial and lateral occipital surfaces (Fig. 3). Cortical stimulation via these subdural electrodes did not elicit any vision-related symptoms. These findings of activa- 

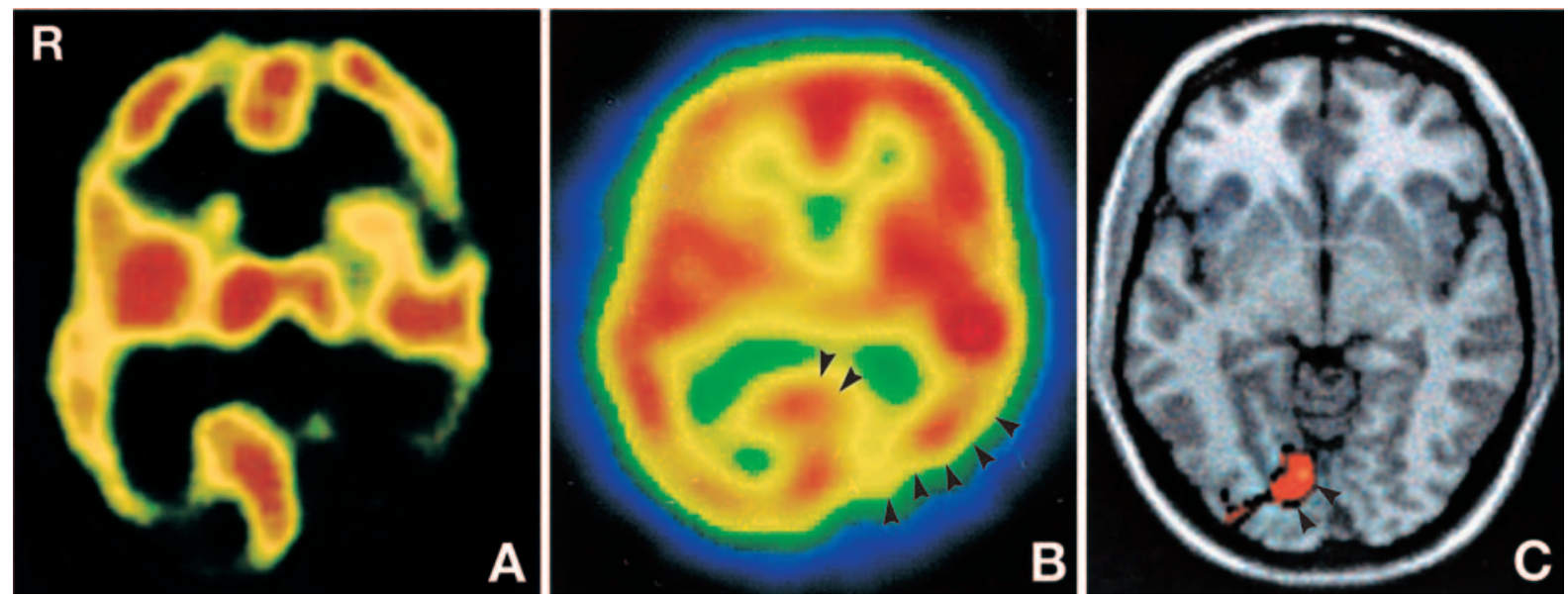

Fig. 2 A, B: Preoperative interictal (A) and ictal (B) single photon emission computed tomography images showing hypoperfusion during the interictal phase and hyperperfusion during the ictal phase in the left occipital and posterior temporal regions (arrowheads). C: Visual activation positron emission tomography image coregistered to the normalized magnetic resonance image showing deactivation in the right occipital cortex (arrowheads).
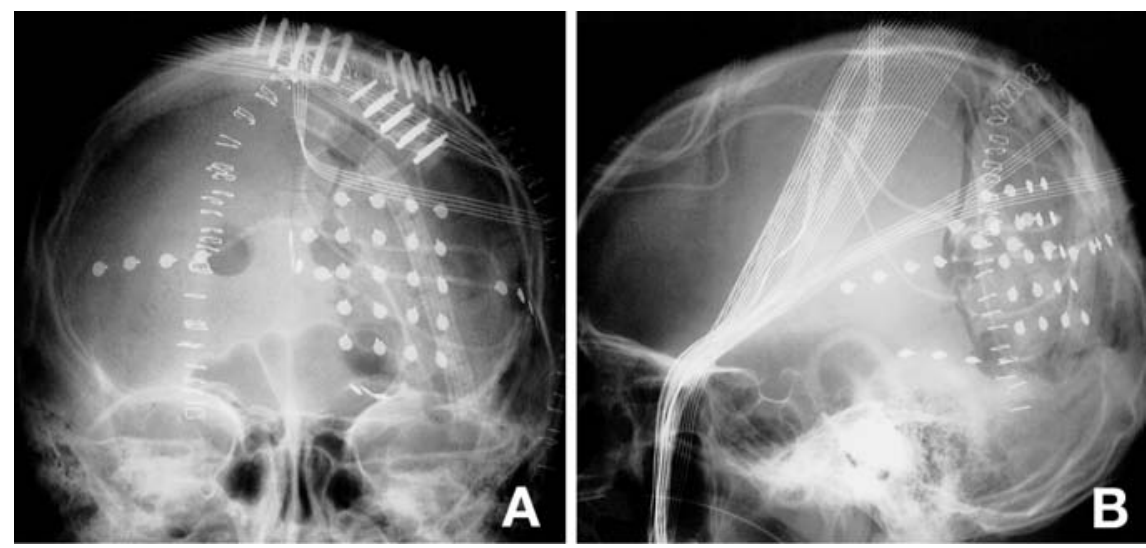

Fig. 3 Anteroposterior (A) and lateral (B) radiographs showing the arrangement of the subdural electrodes.

tion PET and cortical stimulation confirmed the absence of visual function in the left occipital lobe. Ictal subdural electrode EEG with video monitoring confirmed that the seizures originated from the left occipital lobe (Fig. 4).

Resection of the epileptogenic zone identified by ictal subdural electrode EEG and left occipital polymicrogyria was performed (Fig. 1B). The histological diagnosis was gliosis, and no abnormalities specific to focal cortical dysplasia were identified. Postoperatively, the patient became seizure-free and did not report any vision-related symptoms or new visual field impairments.

\section{Discussion}

In this case, electrical cortical stimulation and visual activation PET were used as the brain mapping procedures. Functional MR imaging is another attractive tool for functional brain mapping, but was not applicable to this patient. Electrical stimulation mapping relies on local disruption or activation of cortical circuits and is expected to reflect neuronal activity. In the absence of afterdischarges, this technique has a spatial resolution of 5 to $10 \mathrm{~mm}$ and a temporal resolution of a few seconds. ${ }^{5)}$ Recently, the detailed functional organization of the human visual cortex was clarified with the cortical stimulation technique. ${ }^{9,10}$ ) Activation PET using $\mathrm{H}_{2}{ }^{15} \mathrm{O}$ tracer reflects the regional $\mathrm{CBF}$, which presumably indicates neuronal activation. The correlation between visual function and striatal structure was demonstrated by a visual activation PET study in normal volunteers, and recent developments in this tech- 

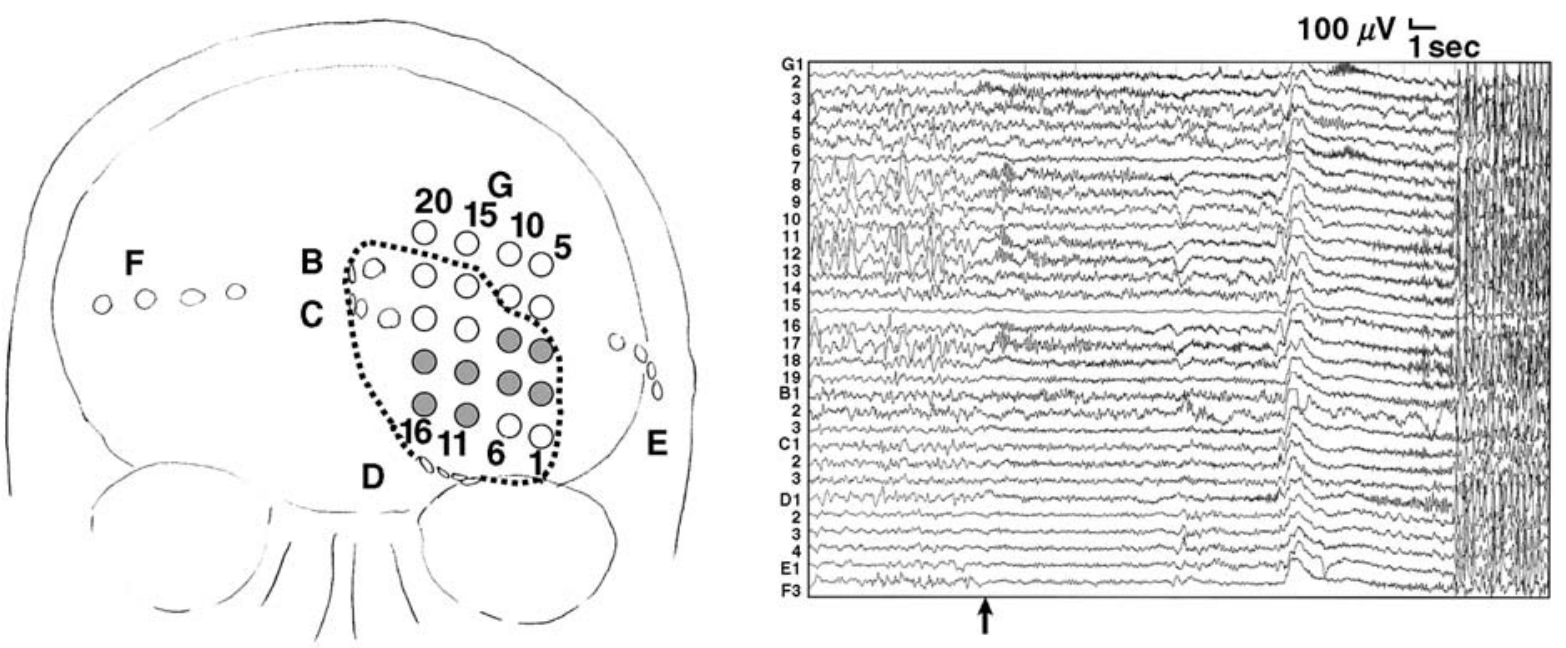

Fig. 4 left: Schematic illustration showing the seizure onsets and resected area. circles: Electrodes, circles in gray: seizure onset, area surrounded by broken line: resected area. right: Ictal encephalograms recorded with the subdural electrodes (A2-reference montage) showing fast activity arose from the left occipital pole $(2,3,7,8,11,12,16$, and 17) and spread to the adjacent areas (electrodes B, C, and D). arrow: Seizure onset.

nique have enabled functional maps to be obtained noninvasively for individual patients. ${ }^{3,5,17)}$ Until recently, the gross spatial resolution of this technique had restricted clinical applications but the spatial resolution has been greatly improved by coregistration with MR images. In the present case, the loss of visual function in the occipital region ipsilateral to the lesion was suggested preoperatively with this mapping technique.

The extent of visual field impairments in children is difficult to assess with conventional perimetry because of the difficulty in obtaining stable fixed vision. However, our patient showed normal visual fields as determined by the confrontation test, so the possibility of functional reorganization had to be considered in the absence of visual function in the left occipital cortex. Preoperative functional mapping with activation PET and cortical stimulation studies confirmed the absence of visual function in the left polymicrogyric occipital lobe. Interestingly, the visual activation PET study showed no activation in the bilateral occipital lobes and deactivation only in the right occipital lobe. In previous studies, the increase of regional CBF was minimum with $1.0-\mathrm{Hz}$ low-frequency flash light stimulation, and deactivation of the visual cortex was induced by involuntary ocular oscillations such as nystagmus and opsoclonus. ${ }^{414)}$ Although the mechanism of deactivation is not yet clear, this phenomenon may be due to temporary downregulation of the visual cortex function triggered by an ocular-motor signal. The deactivation phenomenon may be a physiological mechanism to protect the visual system from inadequate input. ${ }^{14)}$

In our patient, left occipital lobectomy was performed safely and no visual field impairments developed postoperatively. In previous studies of occipital lobe epilepsy, $60 \%$ of patients had visual field deficits before surgery, $32 \%$ of patients developed a new deficit as a result of the surgical resection, and $76 \%$ of patients had complete homonymous hemianopsia after surgery. ${ }^{2,12,16)}$ Functional reorganization of the visual cortex may occur during the development of malformations, such as cortical dysplasia and perinatal ischemic or traumatic insults. ${ }^{8)}$ Only two of 10 patients had visual field abnormalities preoperatively and three developed incomplete visual field impairments after surgery. ${ }^{8)}$ This difference is due to the developmental etiology, since most lesions were acquired in previous series. In cases of developmental malformations, visual function may shift from the occipital region to another area such as the ipsilateral posterior temporal and parietal regions. ${ }^{7)}$ Preoperative surgical planning with functional mapping has become increasingly important in occipital lobe epilepsy surgery to avoid or minimize the chance of developing postoperative visual field impairments. The possibility of functional reorganization has to be considered, particularly in developmental malformations.

The demonstration of convergent results using several techniques such as cortical stimulation and less-invasive methods such as activation PET or functional MR imaging is essential for increasing 
confidence in the functional maps used for clinical decision making. We conclude that functional studies by visual activation PET or functional MR imaging are useful in the preoperative surgical planning and tailored surgery for individual patients with occipital lobe epilepsy.

\section{References}

1) Aykut-Bingol C, Bronen RA, Kim JH, Spencer DD, Spencer SS: Surgical outcome in occipital lobe epilepsy: implications for pathophysiology. Ann Neurol 44: 60-69, 1998

2) Blume WT, Whiting SE, Girvin JP: Epilepsy surgery in the posterior cortex. Ann Neurol 29: 638-645, 1991

3) Fox PT, Mintun MA, Raichle ME, Miezin FM, Allman JM, Van Essen DC: Mapping human visual cortex with positron emission tomography. Nature 323: 806-809, 1986

4) Fox PT, Raichle ME: Stimulus rate determines regional brain blood flow in striate cortex. Ann Neurol 17: 303-305, 1985

5) Fried I, Nenov VI, Ojemann SG, Woods RP: Functional MR and PET imaging of rolandic and visual cortices for neurosurgical planning. J Neurosurg 83: 854-861, 1995

6) Friston KJ, Holmes AP, Worseley KJ, Poline JB, Frith CD, Frackowiak RSJ: Statistical parametric maps in functional imaging: a general linear approach. Hum Brain Mapp 2: 189-210, 1995

7) Kong CK, Wong LY, Yuen MK: Visual field plasticity in a female with right occipital cortical dysplasia. Pediatr Neurol 23: 256-260, 2000

8) Kuzniecky R, Gilliam F, Morawetz R, Faught E, Palmer C, Black L: Occipital lobe developmental malformations and epilepsy: clinical spectrum, treatment, and outcome. Epilepsia 38: 175-181, 1997

9) Lee HW, Hong SB, Seo DW, Tae WS, Hong SC: Mapping of functional organization in human visual cortex: electrical cortical stimulation. Neurology 54: 849-854, 2000
10) Lesser RP, Arroyo S, Crone N, Gordon B: Motor and sensory mapping of the frontal and occipital lobes. Epilepsia 39 (Suppl 4): S69-S80, 1998

11) Rasmussen T: Surgery for epilepsy arising in regions other than the temporal and frontal lobes. Adv Neurol 8: 207-225, 1975

12) Salanova V, Andermann F, Olivier A, Rasmussen $T$, Quesney LF: Occipital lobe epilepsy: electroclinical manifestations, electrocorticography, cortical stimulation and outcome in 42 patients treated between 1930 and 1991. Brain 115: 1655-1680, 1992

13) Talairach J, Tournoux P: Co-Planar Stereotaxic Atlas of the Human Brain. Stuttgart, Thieme-Verlag, 1988, $122 \mathrm{pp}$

14) Wenzel R, Bartenstein P, Dieterich M, Danek A, Weindl A, Minoshima S, Ziegler S, Schwaiger M, Brandt T: Deactivation of human visual cortex during involuntary ocular oscillations. A PET activation study. Brain 119: 101-110, 1996

15) Williamson PD, Spencer SS: Clinical and EEG features of complex partial seizures of extratemporal origin. Epilepsia 27 (Suppl 2): S46-S63, 1986

16) Williamson PD, Thadani VM, Darcey TM, Spencer DD, Spencer SS, Mattson RH: Occipital lobe epilepsy: clinical characteristics, seizure spread patterns, and results of surgery. Ann Neurol 31: 3-13, 1992

17) Zeki S, Watson JD, Lueck CJ, Friston KJ, Kennard C, Frackowiak RS: A direct demonstration of functional specialization in human visual cortex. J Neurosci 11: 641-649, 1991

Address reprint requests to: H. Nakama, M.D., Department of Neurosurgery, National Center Hospital for Mental, Nervous, and Muscular Disorders, National Center of Neurology and Psychiatry, 4-1-1 Ogawahigashi-cho, Kodaira, Tokyo 187-8551, Japan. e-mail:nakama@ncnpmusashi.gr.jp. 